\title{
PRODUCTS OF METHODS OF SUMMABILITY*
}

\author{
BY R. P. AGNEW
}

1. Introduction. Let the transforms

A :

$$
\begin{aligned}
\sigma_{n} & =\sum_{k=1}^{\infty} a_{n k} s_{k}, \\
\tau_{n} & =\sum_{k=1}^{\infty} b_{n k} s_{k},
\end{aligned}
$$

be two regular $\uparrow$ methods of summability. Then the $A$ transform $\left\{\sigma_{n}\right\}$ of the $B$ transform $\left\{\tau_{n}\right\}$ of a sequence $\left\{s_{n}\right\}$ is (if it exists) given by

$$
\sigma_{n}=\sum_{p=1}^{\infty} a_{n p} \tau_{p}=\sum_{p=1}^{\infty} \sum_{k=1}^{\infty} a_{n p} b_{p k} s_{k} .
$$

If $\left\{s_{n}\right\}$, bounded or not, is summable $B$ to $L$ so that $\tau_{n} \rightarrow L$, then regularity of $A$ implies that $\left\{\sigma_{n}\right\}$ exists and $\sigma_{n} \rightarrow L$ as $n \rightarrow \infty$.

If the sequence $\left\{s_{n}\right\}$ is bounded, then the last series in (1) converges absolutely (as a double series) and we can reverse the order of summation to obtain

$$
\sigma_{n}=\sum_{k=1}^{\infty}\left\{\sum_{p=1}^{\infty} a_{n p} b_{p k}\right\} s_{k} .
$$

The matrix $\left\|c_{n k}\right\| \equiv\left\|\sum a_{n p} b_{p k}\right\|$ of (2) is the ordinary matrix product $\left\|a_{n k}\right\|\left\|b_{n k}\right\|$ and the transformation

$A B$ :

$$
\omega_{n}=\sum_{k=1}^{\infty} c_{n k} s_{k}
$$

is denoted by $A B$ as indicated. We shall show that for regular infinite matrices $A, B$, it may not be true that $A B \supset B$; and that regularity of $A, B, D$ and equivalence of $A$ and $D$ do not necessarily imply equivalence of $A B$ and $D B$. We give also related results and applications to kernel transformations.

* Presented to the Society, April 10, 1936.

† The terminology and facts relating to summability which we use are to be found in the expository paper, Report on topics in the theory of divergent series, by W. A. Hurwitz, this Bulletin, vol. 28 (1922), pp. 17-36. 
2. Properties of $A B$. It is easy to see that $A B$ is regular; in fact if $\left\{s_{n}\right\}$, convergent or not, is a bounded sequence summable $B$ to $L$ then we can obtain (2) from (1) and hence conclude that $\left\{s_{n}\right\}$ is summable $A B$ to $L$.

In case $\left\|a_{n k}\right\|$ is row finite (that is, for each $n$ there is an index $k_{n}$ such that $a_{n k}=0$ when $k>k_{n}$ ) the sums over $p$ in (1) and (2) reduce to finite sums; hence we can again obtain (2) from (1) and show that if $\left\{s_{n}\right\}$, bounded or not, is summable $B$ to $L$, then $\left\{s_{n}\right\}$ is summable $A B$ to $L$. Therefore if $\left\|a_{n k}\right\|$ is row finite, $A B \supset B$.

In case $\left\|a_{n k}\right\|$ is not row finite and $\left\{s_{k}\right\}$ is an unbounded sequence summable $B$ to $L$, then the arguments which we used to justify obtaining (2) from (1) and concluding that $\left\{s_{n}\right\}$ is summable $A B$ to $L$ are void. Hence when $\left\|a_{n k}\right\|$ is not row finite, we are unable to prove as above that $A B \supset B$. This inability is fortunate for, as the example of the next section shows, there exist transformations $A^{\prime}$ and $B^{\prime}$ (each of which is not only regular but satisfies in addition several conditions of importance in the theory of summability) and a sequence $\left\{s_{n}^{\prime}\right\}$ summable $B^{\prime}$ to 0 for which the passage from (1) to (2) is impossible. The sequence $\left\{s_{n}^{\prime}\right\}$ is in fact not summable $A^{\prime} B^{\prime}$.

3. An Example. The transformations

$$
\begin{array}{ll}
A^{\prime}: & \sigma_{n}{ }^{\prime}=2^{-1} s_{n+1}+2^{-2} s_{n+2}+2^{-3} s_{n+3}+\cdots, \\
B^{\prime}: & \tau_{n}{ }^{\prime}=\left(1-2^{-n}\right) s_{n}+2^{-n} s_{n+1},
\end{array}
$$

with matrices $\left\|a_{n k}^{\prime}\right\|$ and $\left\|b_{n k}^{\prime}\right\|$, respectively, are regular. The product matrix $\left\|c_{n k}^{\prime}\right\|$ is easily computed, and the transformation $A^{\prime} B^{\prime}$ turns out to be

$A^{\prime} B^{\prime}: \quad \omega_{n}^{\prime}=\left(2^{-1}-2^{-n-2}\right) s_{n+1}+2^{n} \sum_{k=n+2}^{\infty} 2^{-k}\left[1+3 \cdot 2^{-k}\right] s_{k}$.

The sequence $\left\{s_{n}^{\prime}\right\}$ defined by $s_{1}^{\prime}=1$ and the recursion formula $s_{n+1}^{\prime}=-\left(2^{n}-1\right) s_{n}^{\prime},(n=1,2, \cdots)$, is summable $B^{\prime}$ to 0 since substitution of $s_{n}^{\prime}$ for $s_{n}$ in $B^{\prime}$ gives $\tau_{n}^{\prime}=0$ for all $n$. But $\left\{s_{n}^{\prime}\right\}$ is not summable $A^{\prime} B^{\prime}$; in fact $\lim _{k \rightarrow \infty}\left|2^{-k}\left[1+3 \cdot 2^{-k}\right] s_{k}^{\prime}\right|=+\infty$, and the series giving $\omega_{n}^{\prime}$ in terms of $s_{k}$ therefore diverges for every $n$ when $s_{k}=s_{k}^{\prime}$.

Existence of the sequence $\left\{s_{n}^{\prime}\right\}$ summable $B^{\prime}$ but not summable $A^{\prime} B^{\prime}$ implies that the convergence field of $B^{\prime}$ is not con- 
tained in the convergence field of $A^{\prime} B^{\prime}$, and that the relation $A^{\prime} B^{\prime} \supset B^{\prime}$ is false.

4. Further Deductions from the Example. Two methods $A$ and $D$ of summability are called equivalent if each sequence evaluated by either $A$ or $D$ is also evaluated to the same value by the other, that is, $A \supset D$ and $D \supset A$. The transformations $A^{\prime}$ and $I$ (the identity) are equivalent. For $A^{\prime}$ is regular; and it follows from the definition of $A^{\prime}$ that $s_{n+1}=2 \sigma_{n}^{\prime}-\sigma_{n+1}^{\prime}$, so that $\sigma_{n}^{\prime} \rightarrow L$ implies also $s_{n} \rightarrow L$.

Our example shows that regularity of $A, B, D$ together with equivalence of $A$ and $D$ are not sufficient to ensure that $A B$ and $D B$ have the same convergence field; are not sufficient to ensure that $A B \supset D B$; and are not sufficient to ensure equivalence of $A B$ and $D B$. An example to the contrary is obtained by setting $A=A^{\prime}, B=B^{\prime}$, and $D=I$.

5. Kernel Transformations. The matrix transformation $A$ is a special case of the kernel transformation

$A$ :

$$
\sigma(x)=\int_{0}^{\infty} a(x, t) s(t) d t
$$

obtained by putting $s(t)=s_{k}$ when $k-1 \leqq t<k$ and $a(x, t)=a_{n k}$ when $n-1 \leqq x<n, k-1 \leqq t<k$ so that $\sigma(x)=\sigma_{n}$ when $n-1 \leqq x<n$. The $A$ transform of a $B$ transform of a function $s(t)$ is (if it exists) given by

$$
\sigma(x)=\int_{0}^{\infty} d \alpha \int_{0}^{\infty} a(x, \alpha) b(\alpha, t) s(t) d t .
$$

Our example illustrates the fact that, even though $\mathcal{A}$ and $B$ be regular and $s(t)$ be summable $\mathcal{B}$, the formal inversion of order of integration to obtain

$$
\sigma(x)=\int_{0}^{\infty}\left\{\int_{0}^{\infty} a(x, \alpha) b(\alpha, t) d \alpha\right\} s(t) d t
$$

may be unjustified; and that, if $\mathcal{A B}$ denotes the kernel transformation (3), it may in fact fail to be true that $A B \supset B$.

Cornell University 